\section{Valganciclovir for Symptomatic Congenital Cytomegalovirus Disease}

\author{
D.W. Kimberlin, P.M. Jester, P.J. Sánchez, A. Ahmed, R. Arav-Boger, M.G. Michaels, \\ N. Ashouri, J.A. Englund, B. Estrada, R.F. Jacobs, J.R. Romero, S.K. Sood, \\ M.S. Whitworth, M.J. Abzug, M.T. Caserta, S. Fowler, J. Lujan-Zilbermann, \\ G.A. Storch, R.L. DeBiasi, J.-Y. Han, A. Palmer, L.B. Weiner, J.A. Bocchini, \\ P.H. Dennehy, A. Finn, P.D. Griffiths, S. Luck, K. Gutierrez, N. Halasa, \\ J. Homans, A.L. Shane, M. Sharland, K. Simonsen, J.A. Vanchiere, C.R. Woods, \\ D.L. Sabo, I. Aban, H. Kuo, S.H. James, M.N. Prichard, J. Griffin, D. Giles, \\ E.P. Acosta, and R.J. Whitley, for the National Institute of Allergy \\ and Infectious Diseases Collaborative Antiviral Study Group
}

ABSTRACT

\section{BACKGROUND}

The treatment of symptomatic congenital cytomegalovirus (CMV) disease with intravenous ganciclovir for 6 weeks has been shown to improve audiologic outcomes at 6 months, but the benefits wane over time.

\section{METHODS}

We conducted a randomized, placebo-controlled trial of valganciclovir therapy in neonates with symptomatic congenital CMV disease, comparing 6 months of therapy with 6 weeks of therapy. The primary end point was the change in hearing in the better ear ("best-ear" hearing) from baseline to 6 months. Secondary end points included the change in hearing from baseline to follow-up at 12 and 24 months and neurodevelopmental outcomes, with each end point adjusted for central nervous system involvement at baseline.

\section{RESULTS}

A total of 96 neonates underwent randomization, of whom 86 had follow-up data at 6 months that could be evaluated. Best-ear hearing at 6 months was similar in the 6 -month group and the 6-week group ( 2 and 3 participants, respectively, had improvement; 36 and 37 had no change; and 5 and 3 had worsening; $P=0.41$ ). Totalear hearing (hearing in one or both ears that could be evaluated) was more likely to be improved or to remain normal at 12 months in the 6-month group than in the 6 -week group ( $73 \%$ vs. $57 \%, \mathrm{P}=0.01$ ). The benefit in total-ear hearing was maintained at 24 months $(77 \%$ vs. $64 \%, \mathrm{P}=0.04)$. At 24 months, the 6 -month group, as compared with the 6-week group, had better neurodevelopmental scores on the Bayley Scales of Infant and Toddler Development, third edition, on the language-composite component $(P=0.004)$ and on the receptive-communication scale $(P=0.003)$. Grade 3 or 4 neutropenia occurred in 19\% of the participants during the first 6 weeks. During the next 4.5 months of the study, grade 3 or 4 neutropenia occurred in $21 \%$ of the participants in the 6-month group and in $27 \%$ of those in the 6 -week group $(\mathrm{P}=0.64)$.

\section{CONCLUSIONS}

Treating symptomatic congenital CMV disease with valganciclovir for 6 months, as compared with 6 weeks, did not improve hearing in the short term but appeared to improve hearing and developmental outcomes modestly in the longer term. (Funded by the National Institute of Allergy and Infectious Diseases; ClinicalTrials.gov number, NCT00466817.)
The authors' full names, degrees, and affiliations are listed in the Appendix. Address reprint requests to Dr. Kimberlin at the Division of Pediatric Infectious Diseases, University of Alabama at Birmingham, 1600 Seventh Ave. S., Children's Harbor Bldg., Rm. 303, Birmingham, AL 35233, or atdkimberlin@peds.uab.edu.

N Engl J Med 2015;372:933-43. DOI: 10.1056/NEJMoal404599 Copyright $\odot 2015$ Massachusetts Medical Society. 
ONGENITAL CYTOMEGALOVIRUS (CMV) infection is the leading nongenetic cause of sensorineural hearing loss ${ }^{1-4}$ and is the most frequent known viral cause of mental retardation $^{5}$; the infection affects 0.6 to $0.7 \%$ of live births in industrialized countries. ${ }^{6-8} \mathrm{~A}$ total of $10 \%$ of congenitally infected neonates have symptomatic disease at birth, of whom 35\% have sensorineural hearing loss, up to two thirds have neurologic deficits, and 4\% die during the newborn period..$^{7-11}$ Although congenital CMV infection is rare overall, it accounts for $21 \%$ of children with hearing loss at birth and $24 \%$ of those with hearing loss at 4 years of age. ${ }^{1,12}$

The National Institute of Allergy and Infectious Diseases (NIAID) Collaborative Antiviral Study Group (CASG) found that among neonates with symptomatic congenital CMV disease involving the central nervous system (CNS), ganciclovir administered intravenously over a period of 6 weeks was associated with improved audiologic outcomes at 6 months of life, but there was suggestion that this benefit could wane over the first 2 years of life. ${ }^{13}$ Treated infants had fewer developmental delays, according to Denver Developmental evaluations, than untreated infants. ${ }^{14}$ In a follow-up study, the CASG determined the dose of oral valganciclovir (the prodrug of ganciclovir) that results in systemic exposure to ganciclovir that is similar to that with intravenous ganciclovir. ${ }^{15}$ Therapy with intravenous ganciclovir or oral valganciclovir for 6 weeks is now an accepted treatment option for patients with symptomatic congenital CMV disease involving the CNS. ${ }^{16}$

METHODS

STUDY DESIGN AND POPULATION

Neonates with symptomatic congenital CMV disease, with or without CNS involvement, were eligible for enrollment. Given the rarity of this disease, 40 study sites participated, and each was anticipated to contribute only a few study participants. All the study participants had CMV detected in urine or throat-swab specimens by means of culture, shell-vial culture, or polymerase-chainreaction assay. Symptomatic disease was defined as one or more of the following: thrombocytopenia, petechiae, hepatomegaly, splenomegaly, intrauterine growth restriction, hepatitis, or CNS involvement such as microcephaly, intracranial calcifications, abnormal cerebrospinal fluid indexes, chorioretinitis, sensorineural hearing loss, or the detection of CMV DNA in cerebrospinal fluid. Eligible participants had a gestational age of 32 weeks or more, were 30 days of age or less, and weighed at least $1800 \mathrm{~g}$ at the initiation of therapy.

The institutional review board at each study center approved the study protocol. After written informed consent was obtained from the parent or legal guardian, all participants received valganciclovir (at a dose of $16 \mathrm{mg}$ per kilogram of body weight, orally twice daily) for 6 weeks. ${ }^{15}$ Participants then underwent randomization in a 1:1 ratio to receive either continued valganciclovir or placebo for 4.5 months. The dose of the study medication was adjusted monthly for growth. Study drugs (oral valganciclovir and placebo) were provided by Hoffmann-La Roche, which had no role in the study design or data analyses or in the writing of the manuscript or the decision to submit it for publication. Study personnel and the participants' families were unaware of the randomization assignments.

The primary end point prespecified in the protocol was the change in hearing in the better ear ("best-ear" hearing), from baseline to the 6-month follow-up. ${ }^{13}$ Secondary end points prespecified in the protocol included the change in total-ear hearing (i.e., hearing in one or both ears that could be evaluated) from baseline to follow-up at 6,12 , and 24 months; change in best-ear hearing from baseline to follow-up at 12 and 24 months; neurologic impairment at 12 and 24 months; and adverse events leading to the permanent discontinuation of therapy. Tertiary end points included the correlation of viral load in whole blood with audiologic and neurodevelopmental outcomes, adverse events related to the study medication, and characterization of blood concentrations of ganciclovir.

\section{AUDIOLOGIC ASSESSMENTS}

Brain-stem auditory evoked response was assessed at entry, and assessment of brain-stem auditory evoked response or visual-reinforcement audiometry was performed at 6,12 , and 24 months. Hearing thresholds were defined as follows: 0 to $20 \mathrm{~dB}$ for normal hearing, 21 to $45 \mathrm{~dB}$ for mild hearing loss, 46 to $70 \mathrm{~dB}$ for moderate hearing loss, and $71 \mathrm{~dB}$ or higher for severe hearing loss. ${ }^{3,10,13}$ 
An independent audiologist, who was unaware of the randomization assignments, reviewed all the audiometry reports and classified, according to hearing thresholds, all ears that could be evaluated, giving "total ear" classifications. The study audiologist then assigned the "best ear" classification for the participant at that study visit. For example, if a participant had mild hearing loss in the left ear and severe hearing loss in the right ear, the best-ear classification was mild hearing loss. Odd numbers of total ears according to treatment category are reported because a patient may have had only one ear that could be evaluated (e.g., if the patient had otitis media in one ear, such that the ear could not be evaluated, and a normal, second ear that could be evaluated).

\section{OTHER ASSESSMENTS}

The Bayley Scales of Infant and Toddler Development, third edition (Bayley-III), was administered at 12 and 24 months by a neuropsychologist at each study site who was unaware of the randomization assignments. ${ }^{17}$ Whole blood for the evaluation of CMV viral load ${ }^{18}$ was obtained at baseline, weekly for 4 weeks, every 2 weeks for 8 weeks, and monthly for 4 months.

White-cell count, white-cell differential count, measurement of hemoglobin level, platelet count, and aspartate aminotransferase, alanine aminotransferase, total bilirubin, and creatinine measurements were performed serially. Assessments regarding toxic effects were quantified with the use of the NIAID Division of AIDS Toxicity Tables. ${ }^{19}$

\section{STATISTICAL ANALYSIS}

The primary objective was to assess the difference between the 6-week group and 6-month group in the change in best-ear hearing from baseline to 6 months of age. The Wilcoxon-Mann-Whitney test was used for analysis of the primary end point; linear models were used for analysis of secondary end points with adjustment for covariates. We calculated that a sample of 37 participants per group would provide the study with $85 \%$ power to detect an effect size of 0.169 from the null value of 0.5 . We assumed that $15 \%$ of participants would not be eligible for randomization at 6 weeks and that another $10 \%$ would not complete the hearing evaluation at 6 months; therefore, we determined that the original sam- ple should be 94 participants. During the course of the study, the data and safety monitoring board suggested that, owing to inadequate baseline or 6-month data, the sample size should be increased to achieve the targeted 37 participants per group. The sample was increased to 104 participants to accommodate $10 \%$ of the participants with outcomes that could not be evaluated owing to inadequate hearing data at baseline or 6 months. A 5\% overenrollment was allowed for operational purposes.

The modified intention-to-treat population included participants who underwent randomization and received at least one dose of blinded treatment. The prespecified statistical analysis plan dictated that efficacy outcomes be adjusted for CNS involvement. For the secondary audiologic end points, hearing results were analyzed on the basis of two sets of binary outcomes: first, improved hearing or maintenance of normal hearing from baseline to follow-up, as compared with worsened hearing or maintenance of the same degree of hearing loss from baseline to follow-up; and second, worsened hearing from baseline to follow-up, as compared with improved hearing, maintenance of normal hearing, or maintenance of the same degree of hearing loss from baseline to follow-up (see the Supplementary Appendix, available with the full text of this article at NEJM.org, for additional details).

Any hearing assessments completed after cochlear implantation were excluded, as were missing hearing assessments and those that were not able to be evaluated. P values of less than 0.05 for hearing outcomes and less than 0.0071 for neurodevelopmental outcomes were considered to indicate statistical significance. For full details of the study conduct and analyses, see the protocol (including the statistical analysis plan), available at NEJM.org.

\section{RESULTS}

\section{DEMOGRAPHIC CHARACTERISTICS} OF THE PARTICIPANTS

From June 2008 through May 2011, we enrolled 109 participants at 31 study sites. A total of 96 participants were randomly assigned to receive blinded study medication after receiving 6 weeks of valganciclovir; 47 participants were assigned to continue receiving the active drug (the 6-month group), and 49 were assigned to receive placebo 


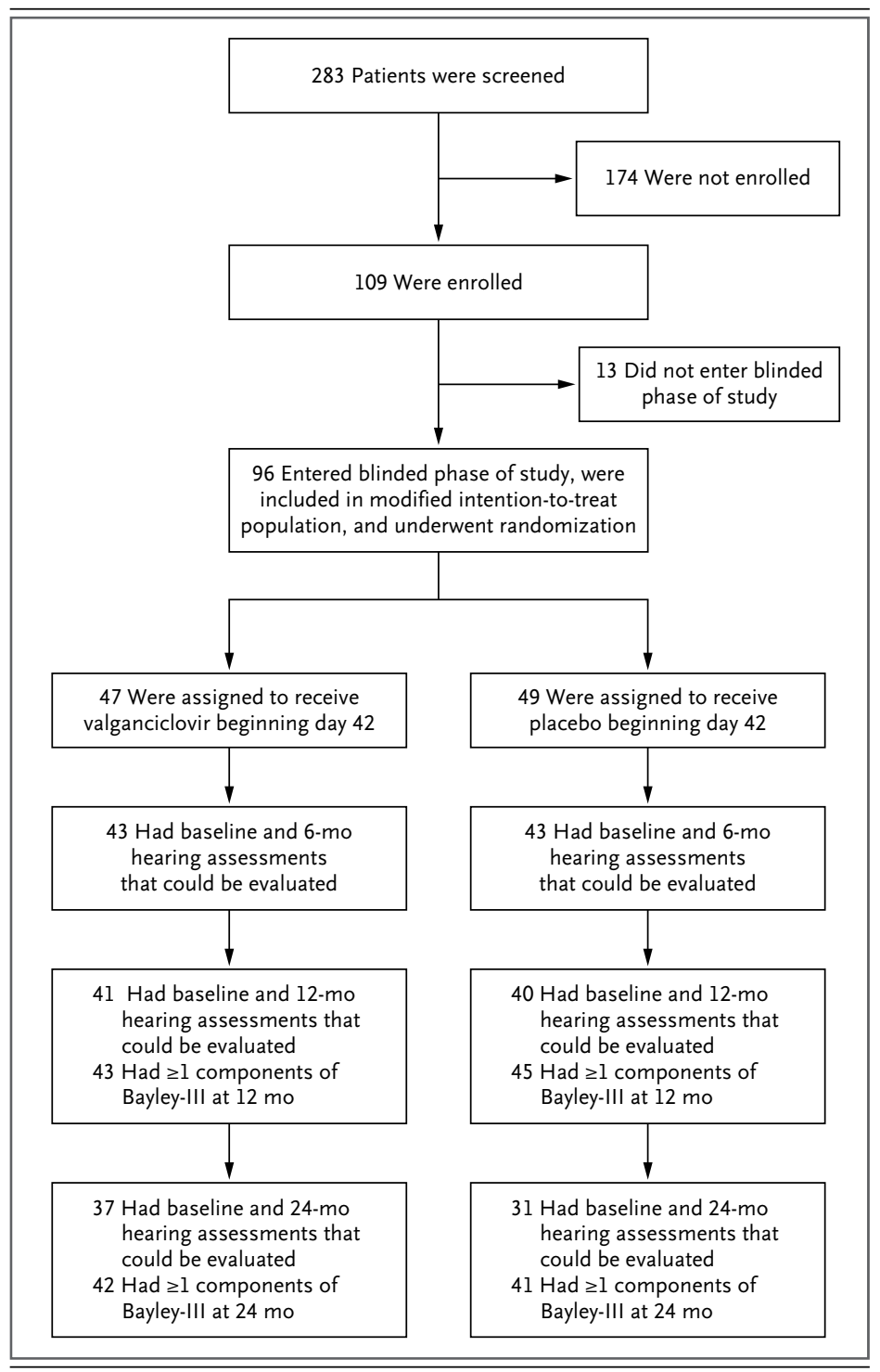

(the 6-week group) (Fig. 1 and Table 1). Of the 96 participants, 9 (6 participants in the 6-month group, and 3 in the 6 -week group) stopped taking the blinded drug before completing 6 months of the study. No participant discontinued the study drug owing to adverse events.

\section{AUDIOLOGIC OUTCOMES Primary End Point}

Among 43 participants in the 6-month group who had assessments that could be evaluated at 6 months, the change in best-ear hearing from baseline to 6 months indicated improvement in 2 participants, no change in 36 , and worsened hearing in 5. Similarly, among 43 participants in the 6-week group, 3 had improved hearing, 37 had
Figure 1. Randomization and Follow-up of the Study Participants.

The participants who had hearing and developmental assessments that could be evaluated at 12 and 24 months of follow-up did not necessarily constitute subgroups of the preceding groups with follow-up assessments. At 6 months, 4 participants in the 6 -month group could not be evaluated because of loss to follow-up ( 2 participants) and incomplete audiologic data (2), and 6 in the 6 -week group could not be evaluated because of loss to follow-up (2), incomplete audiologic data (3), and follow-up assessment that was not performed during the study window (1). At 12 months, 6 participants in the 6-month group could not be evaluated because of cochlear implantation (1 participant), loss to followup (3), and incomplete audiologic data (2), and 9 in the 6-week group could not be evaluated because of cochlear implantation (3), loss to follow-up (1), incomplete audiologic data (3), and follow-up assessment that was not performed during the study window (2). At 24 months, 10 participants in the 6 -month group could not be evaluated because of cochlear implantation (4 participants), loss to follow-up (4), and concurrent otitis media (2), and 18 in the 6 -week group could not be evaluated because of cochlear implantation (6), loss to follow-up (5), incomplete audiologic data (3), follow-up assessment that was not performed during the study window (3), and concurrent otitis media (1). Neurodevelopmental outcomes were assessed with the use of the Bayley Scales of Infant and Toddler Development, third edition (Bayley-III).

unchanged hearing, and 3 had worsened hearing ( $\mathrm{P}=0.41$ by the Wilcoxon-Mann-Whitney test).

\section{Secondary End Points}

In the binary assessment, the change in best-ear hearing from baseline to 6 months was similar in the two treatment groups $(\mathrm{P}=0.24$, after adjustment for baseline CNS involvement) (Table 2). The between-group difference in the change in best-ear hearing from baseline to 12 months and from baseline to 24 months approached significance after adjustment for baseline CNS involvement ( $\mathrm{P}=0.05$ and $\mathrm{P}=0.07$, respectively) (Table 2 ).

In the assessment of total-ear hearing, participants who received 6 months of valganciclovir were more likely than those who received 6 weeks of therapy to have improved hearing or to have maintained normal hearing between baseline and 12 months, after adjustment for CNS involvement at baseline (73\% vs. $57 \%$; odds ratio, 3.04; 95\% confidence interval [CI], 1.26 to 7.35; $\mathrm{P}=0.01$ ) (Table 2). Similar results were evident when prematurity and age at the initiation of treatment were added to the model $(\mathrm{P}=0.01)$. 
Among the 53 participants with baseline CNS involvement, the rate ratio for improved or protected (i.e., maintenance of normal) total-ear hearing at 12 months in the 6-month group, as compared with the 6-week group, was 1.66 (95\% CI, 0.92 to 2.40), and the rate-ratio difference was 0.27 (95\% CI, 0.09 to 0.45 ); for the 28 participants without baseline CNS involvement, the rate ratio was 1.22 (95\% CI, 0.99 to 1.45$)$, and rate-ratio difference was 0.16 (95\% CI, 0.03 to 0.29 ).

The benefit of longer-term therapy in the totalears analysis was maintained at 24 months, with improved outcomes after adjustment for CNS involvement at baseline $(77 \%$ in the 6 -month group vs. 64\% in the 6-week group; odds ratio, 2.61; 95\% CI, 1.05 to 6.43; $\mathrm{P}=0.04$ ) (Table 2). Similar results were evident when prematurity and age at the initiation of treatment were added to the model $(\mathrm{P}=0.04)$. The rate ratio for improved or protected total-ear hearing at 24 months among the 42 participants with baseline CNS involvement in the 6-month group, as compared with the 6-week group, was 1.46 (95\% CI, 0.87 to 2.05), and the rate-ratio difference was 0.23 ( $95 \%$ CI, 0.05 to 0.41 ); among the 26 participants without baseline CNS involvement, the rate ratio was 1.19 ( $95 \% \mathrm{CI}, 0.98$ to 1.40 ), and the rateratio difference was 0.14 (95\% CI, 0.01 to 0.27 ). The timing of initiation of valganciclovir within the first month of life (e.g., $<3$ weeks of age vs. 3 to 4 weeks of age) did not correlate with audiologic outcomes at 12 months or at 24 months ( $\mathrm{P}>0.23$ for both comparisons).

\section{NEURODEVELOPMENTAL OUTCOMES}

In the analysis adjusted for CNS involvement at baseline, participants randomly assigned to receive 6 months of valganciclovir, as compared with those randomly assigned to 6 weeks of treatment, had higher Bayley-III language-composite scores at 24 months $(\mathrm{P}=0.005)$ and higher receptive-communication scale scores at 24 months $(\mathrm{P}=0.003)$. No significant interaction effects were found when outcome and CNS involvement at baseline were incorporated in a single model, indicating that the treatment benefits were similar in the group with CNS involvement and the group without CNS involvement. The differences between the 6-month group and the 6-week group with respect to Bayley-III scores were maintained when age at the initiation of treatment and prematurity were added to the model $(\mathrm{P}=0.004$ and

\begin{tabular}{|c|c|c|c|}
\hline Characteristic & $\begin{array}{l}6 \text { Mo } \\
\text { of Therapy } \\
(\mathrm{N}=47)\end{array}$ & $\begin{array}{l}6 \text { Wk } \\
\text { of Therapy } \\
(\mathrm{N}=49)\end{array}$ & $\begin{array}{c}P \\
\text { Value }\end{array}$ \\
\hline Gestational age - no. (\%) & & & 0.68 \\
\hline 32 to $\leq 37 w k$ & $24(51)$ & $22(45)$ & \\
\hline$>37$ wk & $23(49)$ & $27(55)$ & \\
\hline Age at enrollment — no. (\%) & & & 0.08 \\
\hline$<7$ days & $6(13)$ & $7(14)$ & \\
\hline $7-14$ days & $19(40)$ & $12(24)$ & \\
\hline $15-21$ days & $10(21)$ & $6(12)$ & \\
\hline $22-29$ days & $12(26)$ & $24(49)$ & \\
\hline \multicolumn{4}{|l|}{ Extent of CMV disease — no. (\%)广 } \\
\hline Thrombocytopenia & $38(81)$ & $34(69)$ & 0.24 \\
\hline Petechiae & $22(47)$ & $20(41)$ & 0.68 \\
\hline Hepatomegaly & $26(55)$ & $21(43)$ & 0.31 \\
\hline Splenomegaly & $23(49)$ & $22(45)$ & 0.84 \\
\hline Intrauterine growth restriction & $17(36)$ & $22(45)$ & 0.41 \\
\hline Hepatitis & $21(45)$ & $25(51)$ & 0.55 \\
\hline Central nervous system involvement & $34(72)$ & $29(59)$ & 0.20 \\
\hline Microcephaly — no. (\%) & $14(30)$ & $17(35)$ & 0.19 \\
\hline Chorioretinitis - no. (\%) & $2(4)$ & $1(2)$ & 1.00 \\
\hline $\begin{array}{r}\text { Neuroimaging results - } \\
\text { no./total no. }(\%) \div\end{array}$ & & & 0.62 \\
\hline Normal & $9 / 45(20)$ & $12 / 47(26)$ & \\
\hline Abnormal & $36 / 45(80)$ & $35 / 47(74)$ & \\
\hline $\begin{array}{l}\text { Baseline BSER of the best ear in participants } \\
\text { with } 6 \text {-mo follow-up data - no./ } \\
\text { total no. (\%) } \int\end{array}$ & & & 0.17 \\
\hline Normal & $32 / 43(74)$ & $25 / 43(58)$ & \\
\hline Mild & $5 / 43(12)$ & $8 / 43$ (19) & \\
\hline Moderate & $3 / 43(7)$ & $2 / 43(5)$ & \\
\hline Severe & $3 / 43(7)$ & $8 / 43$ (19) & \\
\hline
\end{tabular}

* Table S1 in the Supplementary Appendix provides a full tabulation of the demographic and clinical characteristics at baseline.

$\uparrow$ Participants could have multiple manifestations of cytomegalovirus (CMV) disease. Hepatitis was defined by an elevated aminotransferase or bilirubin level.

$\$$ Neuroimaging was performed with the use of magnetic resonance imaging, computed tomography, or ultrasonography of the head.

$\int$ Hearing thresholds were assessed with the use of brain-stem auditory evoked response (BSER) and were defined as follows: a threshold of 0 to $20 \mathrm{~dB}$ for normal hearing, 21 to $45 \mathrm{~dB}$ for mild hearing loss, 46 to $70 \mathrm{~dB}$ for moderate hearing loss, and $71 \mathrm{~dB}$ or higher for severe hearing loss.

$\mathrm{P}=0.003$, respectively) (Table 3). All the other components of the Bayley-III assessments trended toward improved outcomes among participants in the 6-month group, as compared with those in the 6-week group. 


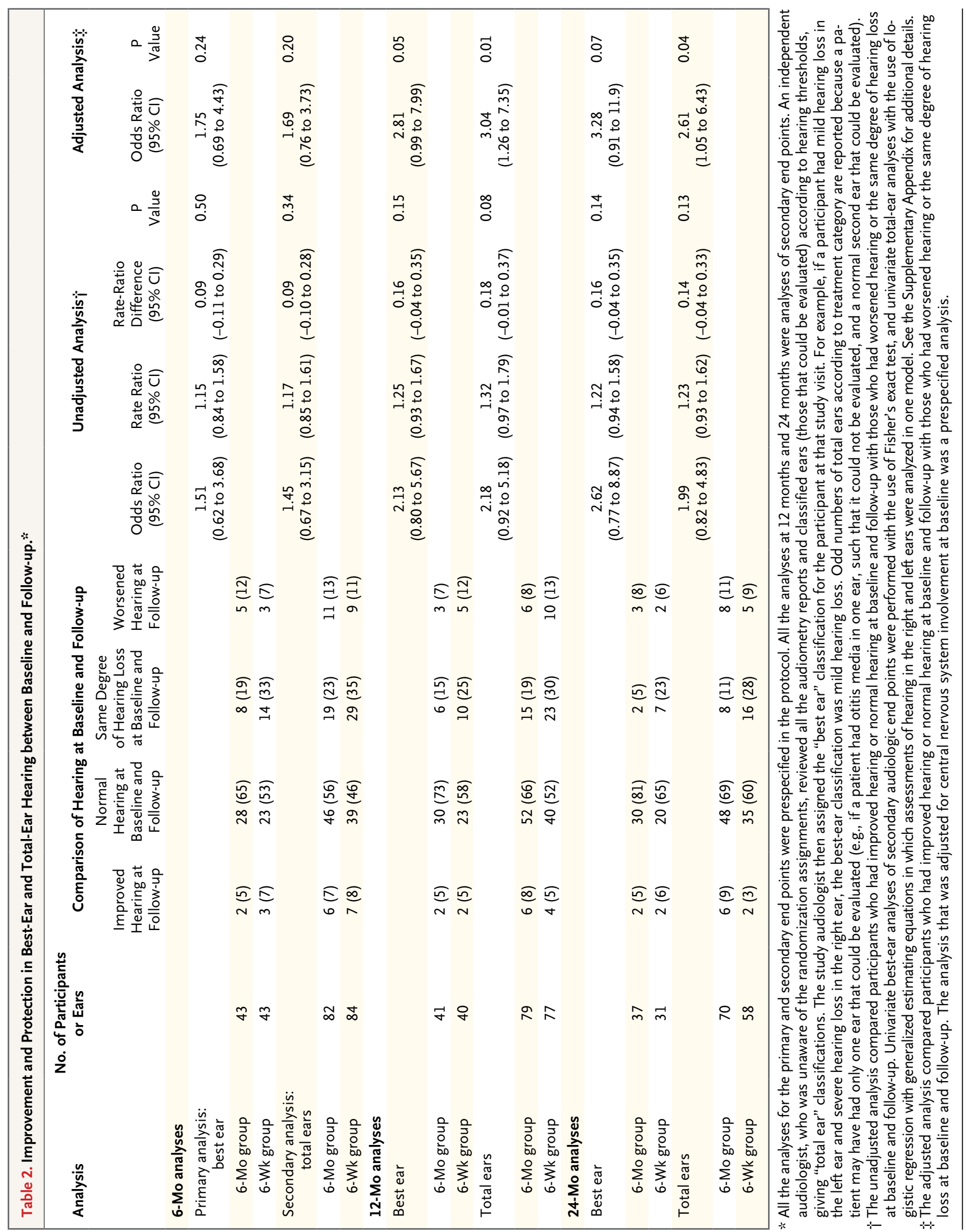




\begin{tabular}{|c|c|c|c|c|c|c|}
\hline \multirow[t]{2}{*}{ Bayley-III Component'í } & \multicolumn{3}{|c|}{ 12-Mo Follow-up } & \multicolumn{3}{|c|}{ 24-Mo Follow-up } \\
\hline & 6-Mo Group & 6-Wk Group & P Value & 6-Mo Group & 6-Wk Group & P Value \\
\hline Cognitive composite & & & 0.01 & & & 0.02 \\
\hline No. of participants & 43 & 45 & & 42 & 41 & \\
\hline Mean & $89.6 \pm 3.0$ & $79.5 \pm 2.8$ & & $84.4 \pm 2.6$ & $76.0 \pm 2.6$ & \\
\hline Range & $55-115$ & $9.5-120$ & & $55-135$ & $55-110$ & \\
\hline Language composite & & & 0.009 & & & 0.004 \\
\hline No. of participants & 41 & 43 & & 41 & 41 & \\
\hline Mean & $87.6 \pm 3.0$ & $76.8 \pm 2.9$ & & $84.6 \pm 2.9$ & $72.5 \pm 2.9$ & \\
\hline Range & $47-118$ & $11-112$ & & $47-121$ & 47-103 & \\
\hline Receptive-communication scale & & & 0.05 & & & 0.003 \\
\hline No. of participants & 41 & 43 & & 41 & 41 & \\
\hline Mean & $7.5 \pm 0.5$ & $6.1 \pm 0.5$ & & $7.3 \pm 0.5$ & $5.2 \pm 0.5$ & \\
\hline Range & $1-14$ & $1-12$ & & $1-14$ & $1-10$ & \\
\hline Expressive-communication scale & & & 0.02 & & & 0.02 \\
\hline No. of participants & 41 & 44 & & 41 & 41 & \\
\hline Mean & $8.0 \pm 0.5$ & $6.5 \pm 0.5$ & & $7.3 \pm 0.5$ & $5.5 \pm 0.5$ & \\
\hline Range & $1-13$ & $1-13$ & & $1-13$ & $1-11$ & \\
\hline Motor composite & & & 0.03 & & & 0.01 \\
\hline No. of participants & 42 & 44 & & 41 & 40 & \\
\hline Mean & $82.6 \pm 3.2$ & $73.2 \pm 3.0$ & & $85.5 \pm 3.3$ & $74.1 \pm 3.2$ & \\
\hline Range & $46-112$ & $11-112$ & & $46-121$ & $46-121$ & \\
\hline Fine-motor scale & & & 0.11 & & & 0.06 \\
\hline No. of participants & 41 & 44 & & 42 & 40 & \\
\hline Mean & $7.3 \pm 0.6$ & $6.0 \pm 0.6$ & & $8.0 \pm 0.6$ & $6.4 \pm 0.6$ & \\
\hline Range & $1-11$ & $0.1-13$ & & $1-15$ & $1-19$ & \\
\hline Gross-motor scale & & & 0.07 & & & 0.02 \\
\hline No. of participants & 42 & 44 & & 42 & 40 & \\
\hline Mean & $6.7 \pm 0.5$ & $5.4 \pm 0.5$ & & $7.0 \pm 0.5$ & $5.3 \pm 0.5$ & \\
\hline Range & $1-14$ & $0.1-15$ & & $1-13$ & $1-12$ & \\
\hline
\end{tabular}

* Plus-minus values are means \pm SE. Data were adjusted for central nervous system involvement at baseline, prematurity, and age at the initiation of treatment. A P value of less than 0.0071 (i.e., 0.05 divided by 7 ) was considered to indicate statistical significance, with the use of Bonferroni adjustment for multiple testing. Ranges represent the minimum and maximum values of the raw data.

$\dagger$ All the composite scores on the Bayley Scales of Infant and Toddler Development, third edition (Bayley-III), range from 40 to 160, with higher scores indicating better developmental outcomes. All the scale scores on the Bayley-III range from 1 to 19, with higher scores indicating better developmental outcomes. Decimal points in the ranges reflect the specific raw data in the scales for at least one study participant, as captured on the case-report form and verified during the on-site monitoring visits.

\section{VIROLOGIC RESULTS}

Viral loads in whole blood decreased in parallel in the two study groups during the first 6 weeks of open-label valganciclovir therapy and then diverged after randomization (Fig. 2). In the analysis that was adjusted for an interaction effect between treatment and the area under the curve (AUC) of the viral load, lower viral loads, as compared with higher viral loads, correlated with better hearing outcomes at 6,12 , and 24 months among participants in the 6-month group $(\mathrm{P}<0.01$ for all comparisons) but not among those in the 


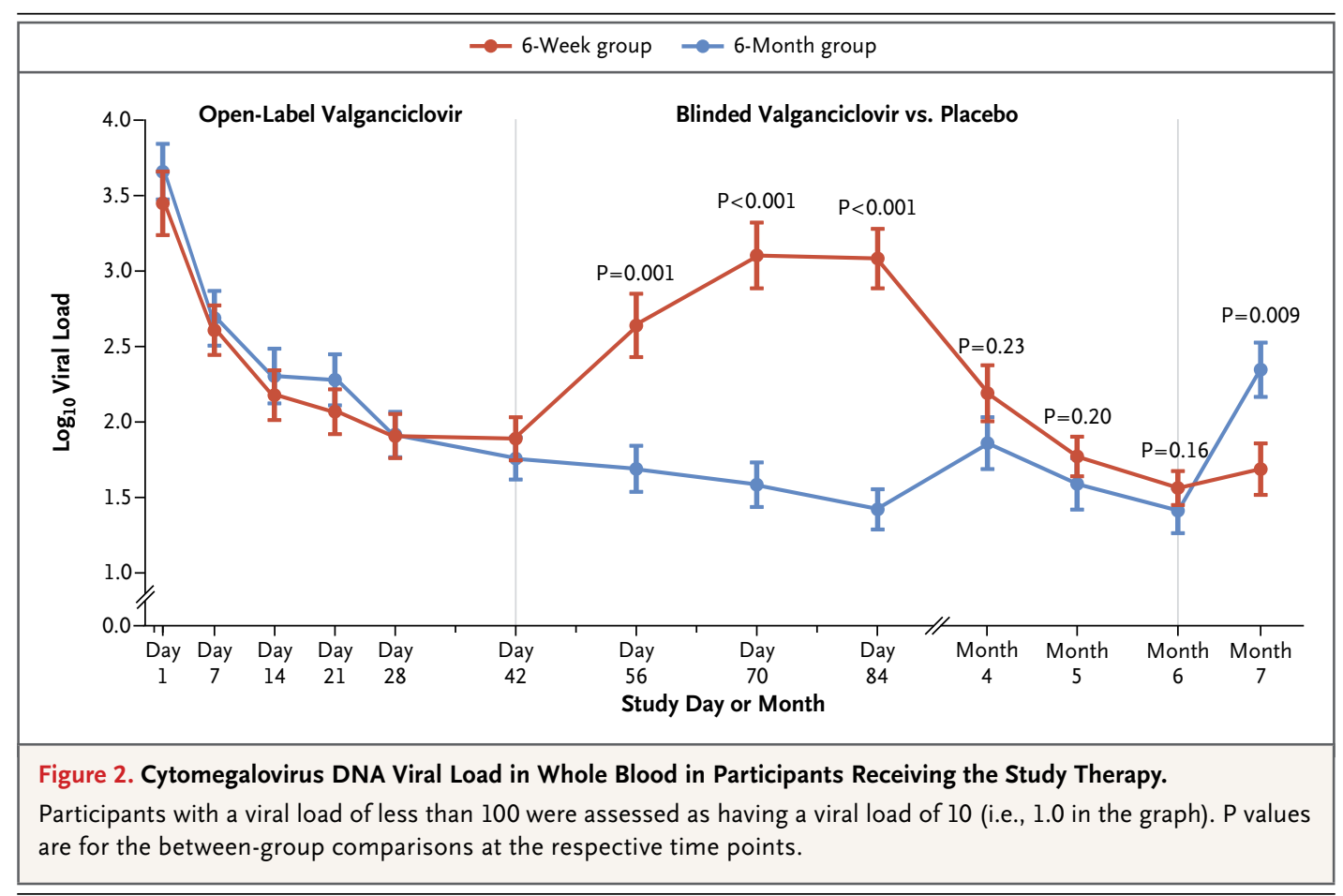

6-week group ( $\mathrm{P}>0.68$ for all comparisons). There was no correlation between the AUC of the viral load and neurodevelopmental outcomes beyond that provided by treatment.

\section{SAFETY ASSESSMENTS}

Of the 109 participants, 21 (19\%) had grade 3 or 4 neutropenia during the first 6 weeks of openlabel valganciclovir therapy. From week 6 through month 6 , a total of 10 of the 47 participants (21\%) who received the active drug had grade 3 or 4 neutropenia, as compared with 13 of 49 (27\%) who received placebo $(\mathrm{P}=0.64)$. A total of 3 participants had the drug temporarily suspended because of an absolute neutrophil count of less than 500 per cubic millimeter. All treatment interruptions occurred within the first 6 weeks of the study, and treatment was resumed after resolution of the neutropenia.

The alanine aminotransferase and aspartate aminotransferase levels increased slightly at months 4 and 5 in the group of participants who received the active drug, although the differences between this group and the group that received placebo were not statistically significant ( $\mathrm{P}>0.59$ for both aminotransferase comparisons) or clinically significant (all mean values, $<90 \mathrm{U}$ per liter). No deaths occurred. There were no significant differences in the rate of adverse events between the two study groups.

\section{DISCUSSION}

We believe that only one randomized, controlled trial of antiviral treatment for symptomatic congenital CMV disease has been conducted previously, also by the NIAID CASG. ${ }^{13}$ Other reports in the literature involve individual cases $^{20-24}$ or small, uncontrolled case series..$^{25-27}$ The earlier randomized, controlled trial showed a benefit of 6 weeks of parenteral ganciclovir therapy with respect to best-ear hearing from baseline to 6 months, but there was a suggestion that this benefit wanes over the first 2 years of life. ${ }^{13}$ On the basis of these data, the change in best-ear hearing from baseline to 6 months was selected as the primary end point of this trial. This selection positioned the current study to extend our knowledge of the effect of antiviral therapy on hearing and allowed for sample-size assessments in the development of the study protocol.

We also selected numerous clinically relevant secondary end points before the initiation of the study to explore the effect of longer-term anti- 
viral treatment on longer-term hearing improvement. These included the change in best-ear hearing and the change in total-ear hearing from baseline to 12 months and from baseline to 24 months in order to more completely ascertain the effect of antiviral treatment on short-term (to 6 months of age) and longer-term (to 2 years of age) time frames. Formal neurodevelopmental outcomes were incorporated as secondary end points in the study to assess the effect of antiviral treatment on neurodevelopmental outcome.

We did not find significant between-group differences in the primary study end point of change in best-ear hearing between baseline and 6 months. The change in total-ear hearing between baseline and 6 months also was similar in the two groups. However, the secondary study end points of change in total-ear hearing between baseline and 12 months and between baseline and 24 months differed significantly between the two groups, with participants receiving 6 months of therapy, as compared with those receiving 6 weeks of treatment, having improved hearing outcomes. Data from our prespecified secondary end points suggest that the 6-month regimen of antiviral treatment modestly improves hearing outcomes in the long term but does not provide an additional benefit with respect to short-term outcomes over that provided by 6 weeks of treatment.

The magnitude of the long-term benefit can be viewed in several ways. The calculation of odds ratios showed that, as compared with patients who received shorter therapy, patients who received longer therapy had 3.0 times the odds of having improved hearing or protection of normal hearing at 12 months and 2.6 times the odds at 24 months. The calculation of the rate ratio showed that among participants with CNS involvement at baseline, those in the 6-month group had a $65 \%$ greater likelihood of having better outcomes from baseline to 12 months than those in the 6 -week group and a $46 \%$ greater likelihood of having better outcomes from baseline to 24 months than those in the 6-week group. Among participants without CNS involvement, the corresponding values were $22 \%$ and $19 \%$. Rate-ratio differences between the groups range from 0.14 to 0.27 , depending on the presence of CNS involvement at baseline and on the follow-up interval. We caution that spurious find- ings may have arisen from the multiple statistical tests conducted for the secondary hearing end points considered in this report.

With respect to neurodevelopmental outcomes, we found that the communicative end points of scores on the language-composite component and the receptive-communication scale of the Bayley-III ${ }^{17}$ assessment, with Bonferroni adjustment for multiple testing, were improved with the longer treatment, with low average results among participants treated for 6 months but borderline results among participants treated for 6 weeks (see the Supplementary Appendix for scoring definitions), after adjustment for factors that could affect development. All the scores on the other components of the Bayley-III were also higher in the 6-month treatment group than in the 6-week group (Table 3), although the differences were not significant. No significant interaction effects were found, indicating similar neurologic treatment benefits regardless of CNS involvement.

The rates of grade 3 or 4 neutropenia during the first 6 weeks of treatment were lower among the participants in the current study who received oral valganciclovir (19\% of participants) than among participants in previous CASG studies who received intravenous ganciclovir for 6 weeks $(63 \%)^{13}$ or intravenous ganciclovir for 2 weeks and oral valganciclovir for 4 weeks (38\%), ${ }^{15}$ perhaps owing to the higher maximum concentration of the drug associated with intravenous versus oral drug delivery. From week 6 to month 7 in the current trial, the incidence of grade 3 or 4 neutropenia was similar among participants randomly assigned to continue valganciclovir and those randomly assigned to receive placebo (21\% and $27 \%$, respectively; $\mathrm{P}=0.64$ ). Thus, druginduced neutropenia is of primary concern during the first 6 weeks of treatment, and the risk appears to be reduced when treatment is solely with oral valganciclovir. ${ }^{13}$ Ganciclovir has toxic effects on the gonads and is carcinogenic in animal models, ${ }^{28}$ and although these toxic effects have not been seen in humans, the information should be conveyed to families of neonates for whom valganciclovir therapy is being considered.

The data from this controlled study suggest that among infants with symptomatic congenital CMV disease, 6 months of oral valganciclovir therapy has a moderately favorable effect on long- 
term audiologic and neurodevelopmental outcomes, after adjustment for baseline CNS involvement; in addition, this regimen was not associated with an excess risk of neutropenia and avoided the need to maintain intravenous access for prolonged periods of time. These data do not apply to infants with asymptomatic congenital CMV infection, since there are no controlled studies showing a benefit in this population and the possibility of harm exists. Since CMV-associated sensorineural hearing loss fluctuates over time in more than one third of patients as part of the natural history of this disease, prospective, controlled trial designs are critical to assess treatment benefit in patients with congenital CMV infections.

Presented in part at the 2013 IDWeek Annual Meeting of the Infectious Diseases Society of America and the Pediatric Infectious Diseases Society, San Francisco, October 2-6, 2013.

Supported by contract N01-AI-30025 from the Division of Microbiology and Infectious Diseases of the National Institute of Allergy and Infectious Diseases.

Disclosure forms provided by the authors are available with the full text of this article at NEJM.org.

APPENDIX

The authors' full names and degrees are as follows: David W. Kimberlin, M.D., Penelope M. Jester, B.S.N., M.P.H., Pablo J. Sánchez, M.D., Amina Ahmed, M.D., Ravit Arav-Boger, M.D., Marian G. Michaels, M.D., M.P.H., Negar Ashouri, M.D., Janet A. Englund, M.D., Benjamin Estrada, M.D., Richard F. Jacobs, M.D., José R. Romero, M.D., Sunil K. Sood, M.D., M. Suzanne Whitworth, M.D., Mark J. Abzug, M.D., Mary T. Caserta, M.D., Sandra Fowler, M.D., Jorge Lujan-Zilbermann, M.D., Gregory A. Storch, M.D., Roberta L. DeBiasi, M.D., Jin-Young Han, M.D., Ph.D., April Palmer, M.D., Leonard B. Weiner, M.D., Joseph A. Bocchini, M.D., Penelope H. Dennehy, M.D., Adam Finn, M.D., Paul D. Griffiths, M.D., Suzanne Luck, M.B., Ch.B., Kathleen Gutierrez, M.D., Natasha Halasa, M.D., James Homans, M.D., M.P.H., Andi L. Shane, M.D., M.P.H., Michael Sharland, M.D., Kari Simonsen, M.D., John A. Vanchiere, M.D., Ph.D., Charles R. Woods, M.D., Diane L. Sabo, Ph.D., Inmaculada Aban, Ph.D., Huichien Kuo, M.S., Scott H. James, M.D., Mark N. Prichard, Ph.D., Jill Griffin, R.N., Dusty Giles, R.N., Edward P. Acosta, Pharm.D., and Richard J. Whitley, M.D.

The authors' affiliations are as follows: the Departments of Pediatrics (D.W.K., P.M.J., S.H.J., M.N.P., J.G., D.G., R.J.W.) and Pharmacology (E.P.A.) and the School of Public Health (I.A., H.K.), University of Alabama at Birmingham, Birmingham, and the University of South Alabama, Mobile (B.E.); University of Texas Southwestern Medical Center, Dallas (P.J.S.), and Cook Children's Medical Center, Fort Worth (M.S.W.) - both in Texas; Carolinas Medical Center, Charlotte, NC (A.A.); Johns Hopkins Medical Institutions, Baltimore (R.A.-B.); Children's Hospital of Pittsburgh of the University of Pittsburgh Medical Center, Pittsburgh (M.G.M., D.L.S.); Children's Hospital of Orange County, Orange (N.A.), Lucile Packard Children's Hospital-Stanford University School of Medicine, Stanford (K.G.), and University of Southern California, Los Angeles (J.H.) - all in California; University of Washington, Seattle (J.A.E.); University of Arkansas for Medical Sciences, Little Rock (R.F.J., J.R.R.); Steven and Alexandra Cohen Children's Medical Center, New York (S.K.S.), University of Rochester Medical Center, Rochester (M.T.C.), and State University of New York, Upstate Medical University, Syracuse (L.B.W.) - all in New York; University of Colorado School of Medicine and Children's Hospital of Colorado, Aurora (M.J.A.); Medical University of South Carolina, Charleston (S.F.); University of South Florida College of Medicine, Tampa (J.L.-Z.); Washington University School of Medicine, St. Louis (G.A.S.); Children's National Medical Center, George Washington University School of Medicine, Washington, DC (R.L.D.); University of Minnesota School of Medicine, Minneapolis (J.-Y.H.); University of Mississippi, Jackson (A.P.); Louisiana State University Health Science Center, Shreveport (J.A.B., J.A.V.); Hasbro Children's Hospital, Alpert Medical School of Brown University, Providence, RI (P.H.D.); Bristol Royal Hospital for Children, Bristol (A.F.), and University College London Medical School (P.D.G., S.L.) and St. George's NHS Trust (M.S.), London — all in the United Kingdom; Vanderbilt University, Nashville (N.H.); Emory University School of Medicine, Atlanta (A.L.S.); University of Nebraska Medical Center, Omaha (K.S.); and University of Louisville School of Medicine, Louisville, KY (C.R.W.).

\section{REFERENCES}

1. Morton CC, Nance WE. Newborn hearing screening - a silent revolution. N Engl J Med 2006;354:2151-64.

2. Fowler KB, McCollister FP, Dahle AJ, Boppana S, Britt WJ, Pass RF. Progressive and fluctuating sensorineural hearing loss in children with asymptomatic congenital cytomegalovirus infection. J Pediatr 1997;130:624-30.

3. Fowler KB, Dahle AJ, Boppana SB, Pass RF. Newborn hearing screening: will children with hearing loss caused by congenital cytomegalovirus infection be missed? J Pediatr 1999;135:60-4.

4. Fowler KB, Boppana SB. Congenital cytomegalovirus (CMV) infection and hearing deficit. J Clin Virol 2006;35:226-31. 5. Elek SD, Stern H. Development of a vaccine against mental retardation caused by cytomegalovirus infection in utero. Lancet 1974;1:1-5.
6. Kenneson A, Cannon MJ. Review and meta-analysis of the epidemiology of congenital cytomegalovirus (CMV) infection. Rev Med Virol 2007;17:253-76.

7. Dollard SC, Grosse SD, Ross DS. New estimates of the prevalence of neurological and sensory sequelae and mortality associated with congenital cytomegalovirus infection. Rev Med Virol 2007;17:355-63.

8. Manicklal S, Emery VC, Lazzarotto T, Boppana SB, Gupta RK. The "silent" globa burden of congenital cytomegalovirus. Clin Microbiol Rev 2013;26:86-102.

9. Williamson WD, Desmond $\mathrm{MM}$ LaFevers N, Taber LH, Catlin FI, Weaver TG. Symptomatic congenital cytomegalovirus: disorders of language, learning, and hearing. Am J Dis Child 1982;136:902-5. 10. Dahle AJ, Fowler KB, Wright JD, Boppana SB, Britt WJ, Pass RF. Longitudinal investigation of hearing disorders in children with congenital cytomegalovirus. J Am Acad Audiol 2000;11:283-90.

11. Boppana SB, Ross SA, Fowler KB. Congenital cytomegalovirus infection: clinical outcome. Clin Infect Dis 2013;57:Suppl 4: S178-S181.

12. Grosse SD, Ross DS, Dollard SC. Congenital cytomegalovirus (CMV) infection as a cause of permanent bilateral hearing loss: a quantitative assessment. J Clin Virol 2008;41:57-62.

13. Kimberlin DW, Lin CY, Sánchez PJ, et al. Effect of ganciclovir therapy on hearing in symptomatic congenital cytomegalovirus disease involving the central nervous system: a randomized, controlled trial. J Pediatr 2003;143:16-25.

14. Oliver SE, Cloud GA, Sánchez PJ, et al. Neurodevelopmental outcomes following ganciclovir therapy in symptomatic congenital cytomegalovirus infections involv- 
ing the central nervous system. J Clin Virol 2009;46:Suppl 4:S22-S26.

15. Kimberlin DW, Acosta EP, Sánchez PJ, et al. Pharmacokinetic and pharmacodynamic assessment of oral valganciclovir in the treatment of symptomatic congenital cytomegalovirus disease. J Infect Dis 2008;197:836-45.

16. Cytomegalovirus infection. In: Pickering LK, Baker CJ, Long SS, Kimberlin DW, eds. Red Book: 2012 report of the Committee on Infectious Diseases. 29th ed. Elk Grove Village, IL: American Academy of Pediatrics, 2012:300-5.

17. Bayley N. Bayley Scales of Infant Development technical manual. 3rd ed. San Antonio, TX: Harcourt Assessment, 2006. 18. Boppana SB, Ross SA, Novak Z, et al. Dried blood spot real-time polymerase chain reaction assays to screen newborns for congenital cytomegalovirus infection. JAMA 2010;303:1375-82

19. National Institute of Allergy and Infectious Diseases, Division of AIDS. Division of AIDS table for grading the severity of adult and pediatric adverse events (DAIDS AE Grading Table). 2004 (http:/l rsc.tech-res.com/Document/safetyand pharmacovigilance/Table_for_Grading _Severity_of_Adult_Pediatric_Adverse _Events.pdf).

20. Meine Jansen CF, Toet MC, Rademaker CM, Ververs TF, Gerards LJ, van Loon AM. Treatment of symptomatic congenital cytomegalovirus infection with valganciclovir. J Perinat Med 2005;33:364-6.

21. Müller A, Eis-Hübinger AM, Brandhorst G, Heep A, Bartmann P, Franz AR. Oral valganciclovir for symptomatic congenital cytomegalovirus infection in an extremely low birth weight infant. J Perinatol 2008;28:74-6.

22. Shoji $\mathrm{K}$, Ito N, Ito $\mathrm{Y}$, et al. Is a 6-week course of ganciclovir therapy effective for chorioretinitis in infants with congenital cytomegalovirus infection? J Pediatr 2010; 157:331-3.

23. Kashiwagi Y, Kawashima H, Nakaji$\mathrm{ma}$ J, et al. Efficacy of prolonged valganciclovir therapy for congenital cytomegalo- virus infection. J Infect Chemother 2011; 17:538-40.

24. Yilmaz Çiftdogan D, Vardar F. Effect on hearing of oral valganciclovir for asymptomatic congenital cytomegalovirus infection. J Trop Pediatr 2011;57:132-4.

25. Tanaka-Kitajima N, Sugaya N, Futatani $\mathrm{T}$, et al. Ganciclovir therapy for congenital cytomegalovirus infection in six infants. Pediatr Infect Dis J 2005;24:782-5.

26. Lombardi G, Garofoli F, Villani P, et al. Oral valganciclovir treatment in newborns with symptomatic congenital cytomegalovirus infection. Eur J Clin Microbiol Infect Dis 2009;28:1465-70.

27. Amir J, Wolf DG, Levy I. Treatment of symptomatic congenital cytomegalovirus infection with intravenous ganciclovir followed by long-term oral valganciclovir. Eur J Pediatr 2010;169:1061-7.

28. Roche Pharmaceuticals. Valganciclovir package insert (http://www.accessdata.fda .gov/drugsatfda_docs/label/2001/21304lbl .pdf).

Copyright @ 2015 Massachusetts Medical Society. 